\title{
Forensic dentistry case book 9: The "bite mark" that caused confusion
}

SADJ June 2017, Vol 72 no 5 p228 - p229

H Bernitz', BA Kloppers ${ }^{2}$

\section{INTRODUCTION}

A bite mark case was received from the Eastern Cape for analysis in the Department of Oral Pathology and Oral Biology. The evidence included a photograph of a bite mark on the left cheek of the victim, see Figure 1, two silicone impressions of the bite mark and a set of study models of the suspect's dentition. Although the evidence proved to be sufficient for analysis, the recommended protocol for the collection of evidence was not followed. ${ }^{1}$ No American Board of Forensic Pathology (ABFO) rule or metric scale was used during the photographic session and the impression was extremely thin with no backing to maintain anatomical contours of the area bitten.

On examination of the evidence, several highly characteristic features were noted in the bite mark. The most relevant was the shovel shaped maxillary anteriors, showing the characteristic mesial and distal flaring. The other features that were clearly visible were the absence of the 22 and the anterior positioning of the 11 in relation to the 21 . There were no recognisable dental features in the lower arch.

All the above mentioned features were present in the dentition of the suspect. After the macroscopic dental analysis of the bite mark and the suspect's dentition, the case was sent to Silverton Forensic Laboratory for a tool mark and microscopic analysis of the evidence. The physical

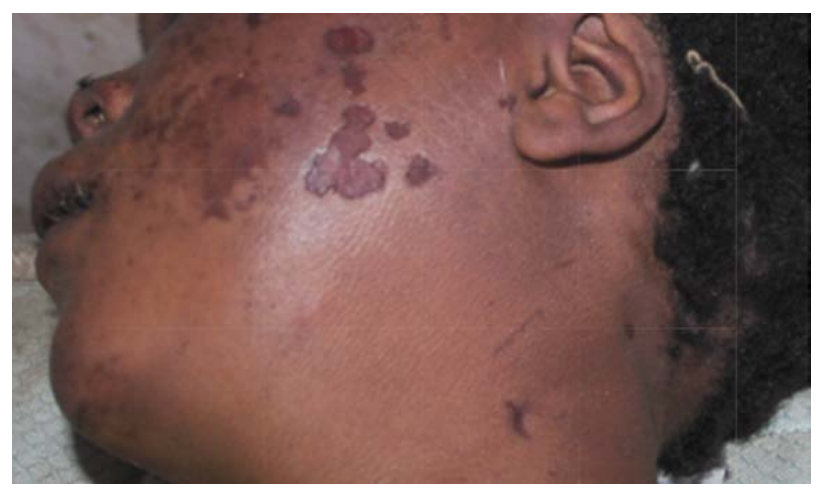

Figure 1: Bite mark present on the left cheek of the victim.

1. Herman Bernitz: BChD., Dip (Odont)., MSc., PhD. (Pret). Department of Oral Pathology and Oral Biology, Faculty of Health Sciences, University of Pretoria.

2. BA Kloppers: South African Police Service, Siverton Forensic Laboratory, Pretoria.

\section{Corresponding author}

\section{Herman Bernitz:}

Department of Oral Pathology and Oral Biology, Faculty of Health

Sciences, University of Pretoria. E-mail: bernitz@iafrica.com match as well as the microscopic analysis showed strong concordance between the suspect's dentition and the bite mark present on the victim. The suspect was thus the most likely initiator of the bite marks found on the body of the deceased. For purposes of the court case, bite registrations of the suspect's dentition were made by pressing the models of the suspect's dentition into a plasticine clay, see Fgures $2 \& 3$.

Two detailed forensic dental reports, one from the each of the two authors, were submitted to the State Advocate for presentation in the High Court.

\section{INTERESTING TURN OF EVENTS.}

The forensic pathologist in the case was subpoenaed to appear several days before our appearance. During cross examination the forensic pathologist stated that the marks present on the left cheek were in fact post-mortem changes and definitely not bite marks. When the pathologist was asked to elaborate on his findings, he pointed to the

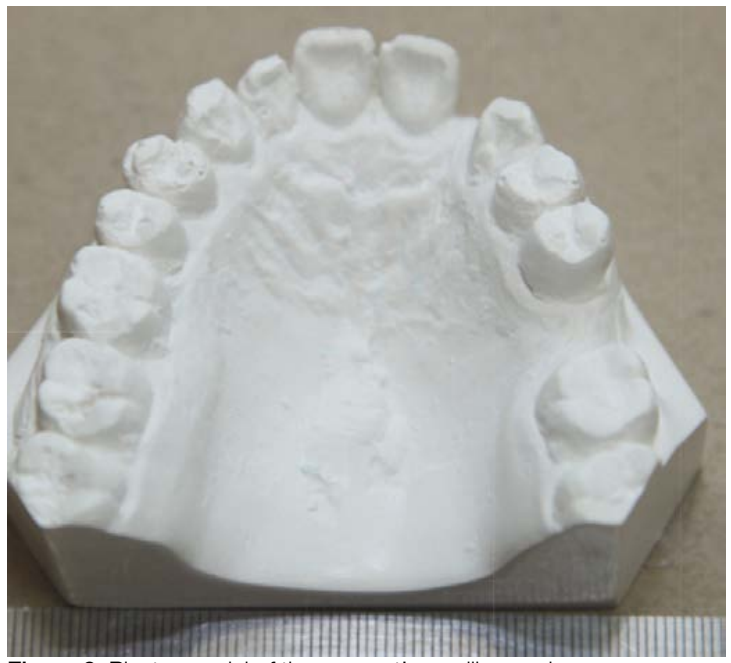

Figure 2: Plaster model of the suspect's maxillary arch.

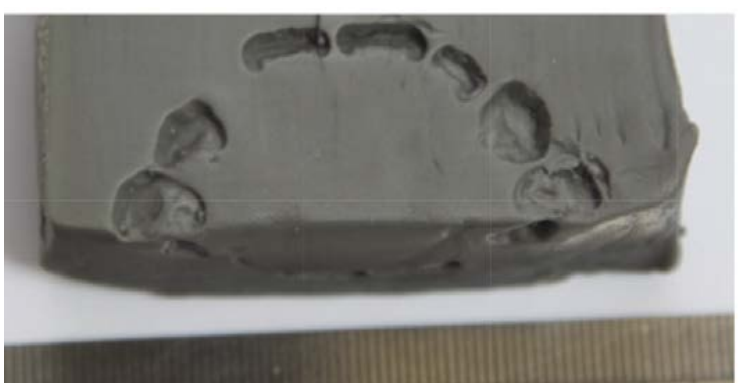

Figure 3: Bite marks of suspect's dentition in the Plasticine clay made by pushing the maxillary model into plasticine clay. 


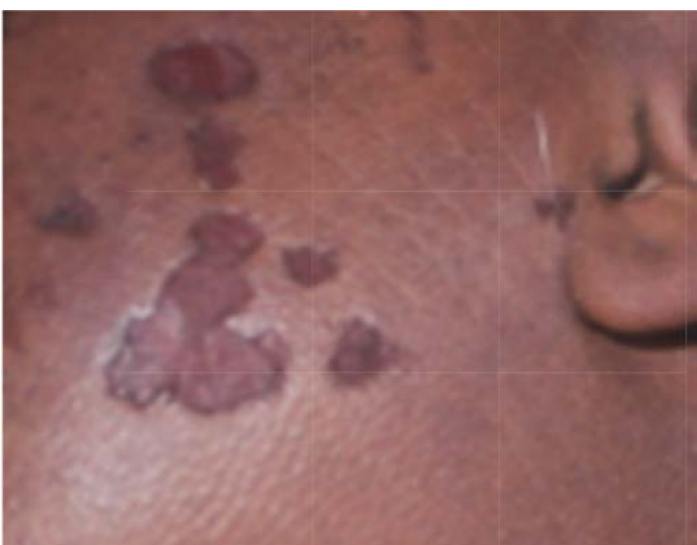

Figure 4: Cropped image of forensic pathologist's "bite mark"

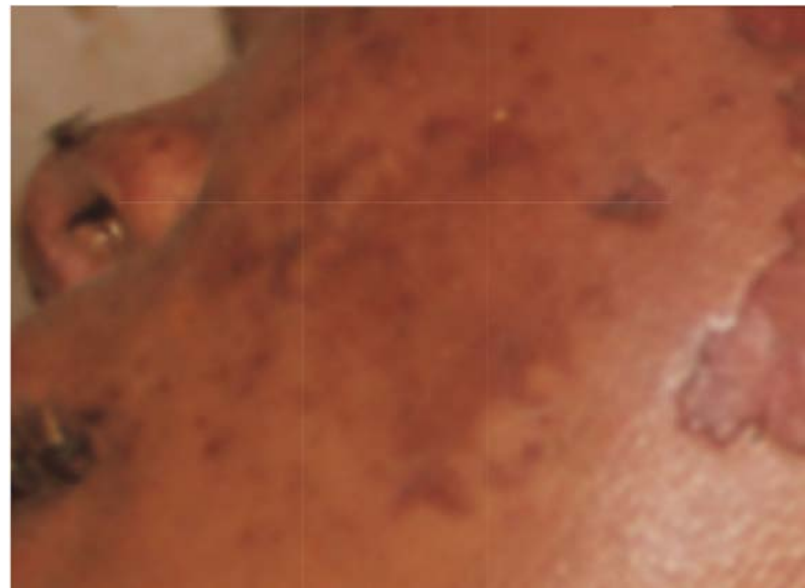

Figure 5: Cropped image of the real bite mark.

marks posterior to the bite mark, misinterpreting them to be the bite marks described in our report, see Figure 4. The pathologist went into great detail describing how the marks could not be bite marks, considering the shape and size of the marks.

He was absolutely correct, as he was not describing the correct set of marks! The judge and state advocate were very relieved when we showed them that the marks pointed out by the forensic pathologist were in fact not the bitemarks inflicted by the suspect, but posterior to the real bite mark which the pathologist had overlooked, see Figure 5.

\section{CONCLUSION}

This case highlights the fact that only bite mark experts should examine bite marks. ${ }^{2}$ In his attempts to discredit our evidence, the forensic pathologist had homed in on the incorrect marks, present in the same area as the real bite marks. The bite mark evidence presented to the High Court was accepted and it can be reported that the suspect received two life sentences, one for rape and one for murder.

The names and places have been changed to protect the identities of the victim and of the forensic pathologist. Permission has been granted by the Department of Justice to present this information, as it is no longer sub judice.

\section{References}

1. Bernitz $H$, van Niekerk PJ. Bungled bite mark evidence collection: a protocol for the prevention thereof. J South African Dental Ass 2003: 58(1); 18-21.

2. Bernitz $H$, Owen JH, Van Heerden WFP, Solheim T. An integrated technique for the analysis of skin bite marks. J For Sci 2008:53(1) 194-8.

\section{new \\ STRONGE \\ STRPAIR} WU \&EPAIR POWERED BY NOVAMIN

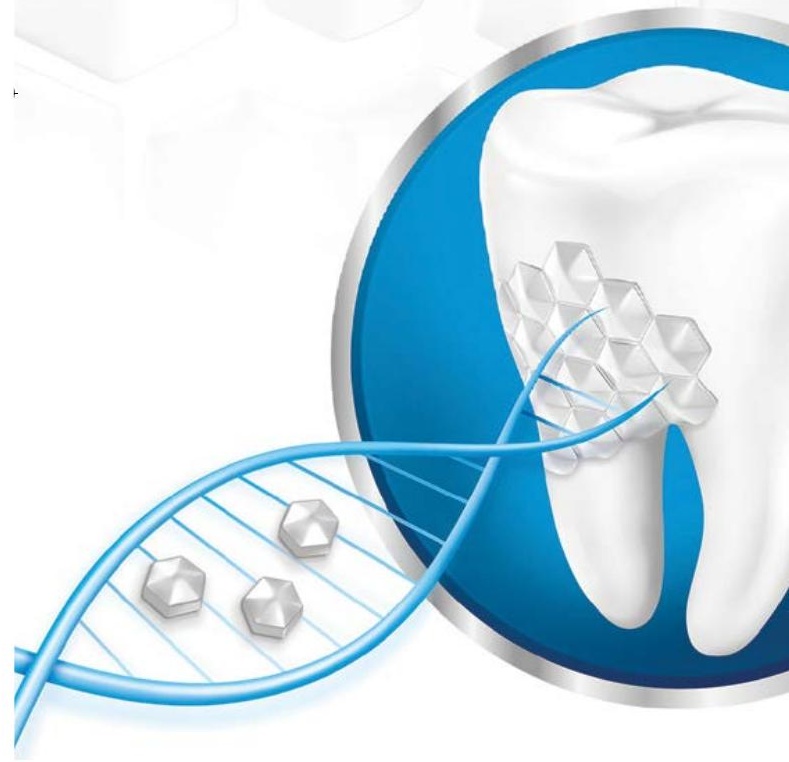

